\title{
An easy nomogram to predict 30 -day mortality in warfarin overdose patients undergoing endoscopy for gastrointestinal bleeding
}

\author{
Datice Tolunay, ${ }^{1}$ (D) Aziz Ahmet Surel ${ }^{2}$ \\ 'Department of Cardiology, Ankara Gulhane Training and Research Hospital, Ankara, Turkey \\ ${ }^{2}$ Department of General Surgery, Ankara City Hospital, Ankara, Turkey
}

\begin{abstract}
Introduction: Gastrointestinal bleeding (GIB) is a crucial medical issue in patients receiving oral anticoagulant therapy. Thus, the management of these patients is important in daily clinical practice. In this study, first, we sought to show the predictors of mortality and, second, provide a nomogram for clinicians to evaluate the risk of mortality in patients undergoing endoscopic therapy with warfarin overdose.
\end{abstract}

Materials and Methods: Patients who underwent endoscopic treatment with warfarin overdose and GIB between February 15, 2019, and March 20, 2021, were retrospectively evaluated. Clinical, demographic, and laboratory parameters of patients were recorded. The primary outcome was 30 -day all-cause mortality after the procedure.

Results: A total of 359 patients admitted with warfarin overdose and GIB who underwent endoscopic treatment were included in the study. All-cause death was observed in $50(14 \%)$ patients in the 30-day period after the procedure. According to univariate and multivariate logistic regression analysis, age $(\mathrm{OR}=1.019$; 95\% $\mathrm{Cl}=1.000-1.039 ; \mathrm{p}=0.008$ ), hypertension (OR=1.909; $95 \% \mathrm{Cl}=1.051-3.468 ; \mathrm{p}=0.004)$, alcohol history $(\mathrm{OR}=1.618 ; 95 \% \mathrm{Cl}=1.196-2.954 ; \mathrm{p}=0.018)$, and albumin value $(\mathrm{OR}=0.318 ; 95 \% \mathrm{Cl}=0.214-0.471 ; \mathrm{p}=0.001)$ were determined as independent predictors for 30-day all-cause mortality. The areas under the curves of the nomogram were 0.73 (95\% Cl: $0.70-0.76)$ may have clinical usefulness.

Conclusion: This study provides a nomogram containing age, hypertension, alcohol, and albumin that can be conveniently used to predict individual mortality in warfarin overdose patients undergoing endoscopy for GIB. Keywords: Gastrointestinal bleeding, mortality, overdose, warfarin

\section{Introduction}

Oral anticoagulant (OAC) treatment is a vital treatment method for the prophylaxis and treatment of thromboembolic diseases, which constitute a wide spectrum in terms of localization and clinic. ${ }^{[1]}$ However, the maintenance dose required for the therapeutic target, the International Normalized Ratio (INR) value, varies between individuals. Warfarin overdose is relatively rare, but it is associated with significant morbidity potential. ${ }^{[2]}$ Bleeding is a common complication and is the most important reason 
limiting the widespread use of warfarin. ${ }^{[3]}$ Therefore, it is important to achieve an effective INR with a low risk of bleeding. Gastrointestinal bleeding (GIB) due to warfarin overdose is a common complication with a rate of $4.5 \% /$ year. ${ }^{[4]}$ For patients with unknown gastric lesions, even therapeutic INR values may cause GIB. Studies have shown that besides environmental factors, genetic factors may play a role in bleeding complications. ${ }^{[5]}$ Data to guide the optimal warfarin management after admission with GIB are limited. ${ }^{[6,7]}$

Identifying clinical factors and laboratory parameters affecting mortality in patients presenting with GIB after warfarin overdose may be an important way to improve clinical results. ${ }^{[9,10]}$ Determining the current risk with the scales determined in patients using warfarin is an important parameter that can affect the duration and management of treatment in patients with GIB and can predict the risk of mortality. In patients with high-risk scores, closer INR follow-up can be planned.

In the light of the above information, we, therefore, aimed to determine the predictors of mortality in patients who undergo endoscopic treatment with warfarin overdose and GIB and to develop a nomogram that can be used as a mortality predictor in these patients.

\section{Materials and Methods}

Patients who underwent endoscopic treatment with warfarin overdose and GIB between February 15, 2019, and March 20, 2021, were retrospectively evaluated. Patients who received antiplatelet therapy other than warfarin, which may affect the risk of bleeding and the clinical results of the patient, and patients who experienced procedural complications during endoscopy were excluded from the study. The clinical and demographic data of the patients and laboratory parameters on admission to the hospital were recorded. Age, gender, hypertension, diabetes mellitus, coronary artery disease, previous cerebrovascular disease, chronic renal failure, cancer history of smoking and alcohol use, admission INR, and serum albumin values of the patients were recorded. The primary outcome was 30-day mortality after the endoscopy date which defined from hospital records and the database of the Republic of Turkey Ministry of Health. The patients clinical and laboratory data were presented according to two groups as survivors and non-survivors. The study was approved by the Ankara City Hospital Ethics Committee (Date: 04/07/2021, Decision no: E2-21-424).

\section{Statistical Analysis}

All statistical analyses were done using by Stata (version 16.0 MP; StataCorp). The distribution of continuous variables was determined using the Kolmogorov-Smirnov test. Continuous data were defined as mean \pm SD for normal distributions and as median (range) for skewed distributions. Categorical data were defined as the number of cases. Statistical analysis differences in variables showing normal distribution were compared between the two groups using Student's t-test, Mann-Whitney U-test was used for non-normally distributed data. Categorical variables were compared using Pearson's Chisquare test. To show significant predictors of 30-day allcause of mortality, a univariate logistic regression model was created for each variable, and then those which had $<0.1 \mathrm{P}$-values were tested in the multivariable logistic regression model. Odds ratios (ORs) and their 95\% Confidence intervals (CIs) for mortality were presented. Receiver operating characteristic (ROC) analysis was used to show the discrimination of the final model. Finally, a nomogram including significant predictors was graphed. $\mathrm{P}<0.05$ was considered significant in all statistical analyzes.

\section{Results}

A total of 359 patients admitted with warfarin overdose and GIB who underwent endoscopic treatment were included in the study. The number of female patients was 194 (54.0\%). Hypertension in 211 (59.8\%) patients, diabetes in 72 (20.4\%), coronary artery disease in 136 (38.7\%), cerebrovascular event in $92(26.1 \%)$, chronic renal failure in 21 (5.9\%), and $17(4.8 \%)$ had a history of cancer. The number of smoking patients was $183(51.0 \%)$, and the number of patients using alcohol was 56 (15.6\%).

All-cause death was observed in 50 (14\%) patients in the 30-day period after the procedure. Between the survivors and non-survivors groups, the mean age was significantly higher in the non-survivors group ( $\mathrm{p}=0.007)$. Hypertension was significantly higher in the non-survivors group $(p=0.003)$. Although alcohol use was more common in the non-survivors group, the difference was not significant $(p=0.078)$. There were no statistically significant differences between survivors and non-survivors groups for gender $(\mathrm{p}=0.76)$, diabetes mellitus $(\mathrm{p}=0.76)$, coronary artery disease $(\mathrm{p}=0.90)$, cerebrovascular disease $(\mathrm{p}=0.44)$, chronic renal failure $(\mathrm{p}=0.57)$, history of cancer $(\mathrm{p}=0.62)$, and smoking $(\mathrm{p}=0.29)$.

Most patients (45.0\% $\mathrm{n}=159)$ had dysrhythmia for the in- 
dication of anticoagulation. Subsequently, 87 (24.6\%) patients had valvular heart disease, $45(12.7 \%)$ patients had cerebrovascular disease, 44 (12.5\%) patients had pulmonary thromboembolism, and 18 (5.1\%) patients had venous thromboembolism for anticoagulation indications. There was no significant difference between the survivors and non-survivors groups in terms of warfarin indications $(\mathrm{p}=0.39)$. All demographic and clinical characteristics of the patients according to survivors and non-survivors groups are shown in Table 1.

The mean INR value at admission was $9.612 \pm 5.287$ for all patients, 9.442 \pm 5.178 in the survivors group, $10.660 \pm 5.867$ in the non-survivors group, and there was no significant difference between the groups $(\mathrm{p}=0.13)$. Serum albumin levels were on average $3.61 \pm 0.62$ for all patients, $3.66 \pm 0.57$ in the survivors group, and $3.34 \pm 0.81$ in the non-survivors group, the difference was significant ( $\mathrm{p}=0.002$ ), (Fig. 1). Upper GIB was found in $220(61.2 \%)$ of the patients, and lower GIB in 139 (38.3\%). Upper GIB was significantly higher in the non-survivors group ( $\mathrm{p}=0.001)$. The baseline clinical characteristics of the patients according to survival status are summarized in Table 2.

Univariate and multivariate logistic regression analysis were used to identify factors predicting 30-day mortality. Age (OR=1.019; 95\% CI=1.000-1.039; $\mathrm{p}=0.008)$, hypertension ( $\mathrm{OR}=1.909 ; 95 \% \mathrm{CI}=1.051-3.468 ; \mathrm{p}=0.004)$, alcohol (OR=1.618; 95\% Cl=1.196-2.954; $\mathrm{p}=0.018)$, and

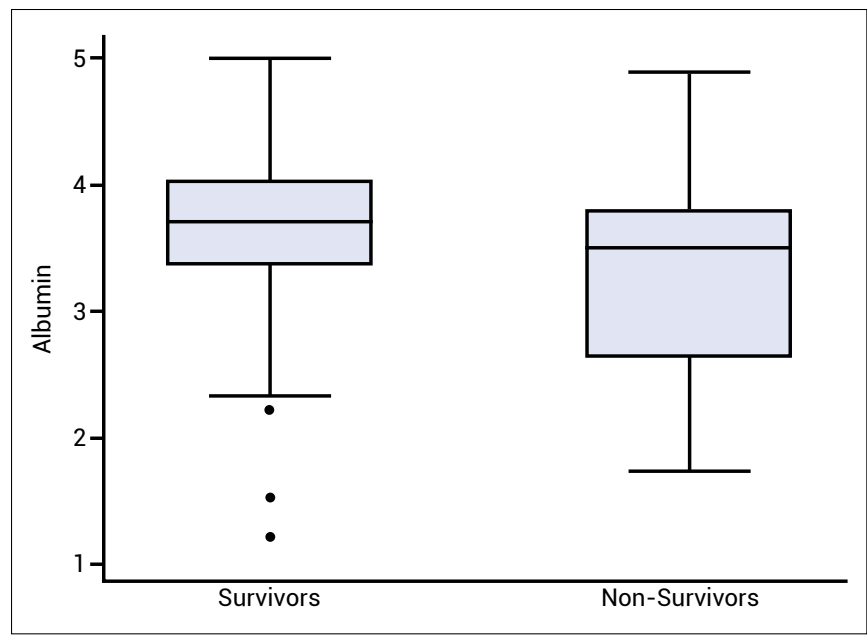

Figure 1. The box plot for serum albumin levels according to the survivors and non-survivors groups.

serum albumin value $(\mathrm{OR}=0.318 ; 95 \% \mathrm{Cl}=0.214-0.471$; $\mathrm{p}=0.001$ ) were determined as independent predictors for 30-day all-cause mortality. Univariate and multivariate logistic regression analysis data are presented in Table 3.

The accuracy of predicting mortality was assessed by the area under the ROC curve which was $=0.73$ (an acceptable discrimination) as shown in Figure 2. A novel nomogram has been developed with significant predictors (age, hypertension, alcohol, and serum albumin) for the primary outcome and was graphed in Figure 3.

\section{Table 1. Baseline demographic of patients according to the survivor status}

\begin{tabular}{|c|c|c|c|c|c|c|c|}
\hline & \multicolumn{2}{|c|}{$\begin{array}{c}\text { Total } \\
\mathrm{n}=359\end{array}$} & \multicolumn{2}{|c|}{$\begin{array}{l}\text { Survivors } \\
n=309\end{array}$} & \multicolumn{2}{|c|}{$\begin{array}{c}\text { Non-survivors } \\
\mathrm{n}=50\end{array}$} & \multirow[t]{2}{*}{$\mathbf{p}$} \\
\hline & $\mathbf{n}$ & $\%$ & $\mathbf{n}$ & $\%$ & $\mathbf{n}$ & $\%$ & \\
\hline Age, mean (SD) & \multicolumn{2}{|c|}{$70(13)$} & \multicolumn{2}{|c|}{$69(14)$} & \multicolumn{2}{|c|}{$75(11)$} & 0.007 * \\
\hline \multicolumn{8}{|l|}{ Gender } \\
\hline Female & 194 & 54.0 & 166 & 53.7 & 28 & 56.0 & 0.76 \\
\hline Male & 165 & 46.0 & 143 & 46.3 & 22 & 44.0 & \\
\hline \multicolumn{8}{|l|}{ History of disease } \\
\hline Hypertension & 211 & 59.8 & 173 & 56.7 & 38 & 79.2 & $0.003 *$ \\
\hline Diabetes mellitus & 72 & 20.4 & 63 & 20.7 & 9 & 18.8 & 0.76 \\
\hline Coronary artery disease & 136 & 38.7 & 117 & 38.6 & 19 & 39.6 & 0.90 \\
\hline Cerebrovascular disease & 92 & 26.1 & 76 & 24.9 & 16 & 33.3 & 0.44 \\
\hline Chronic renal failure & 21 & 5.9 & 19 & 6.2 & 2 & 4.2 & 0.57 \\
\hline Cancer & 17 & 4.8 & 14 & 4.6 & 3 & 6.3 & 0.62 \\
\hline Smoking & 183 & 51.0 & 161 & 52.1 & 22 & 44.0 & 0.29 \\
\hline Alcohol & 56 & 15.6 & 44 & 14.2 & 12 & 24.0 & 0.078 \\
\hline
\end{tabular}


Table 2. Baseline clinical characteristics of patients according to the survivor status
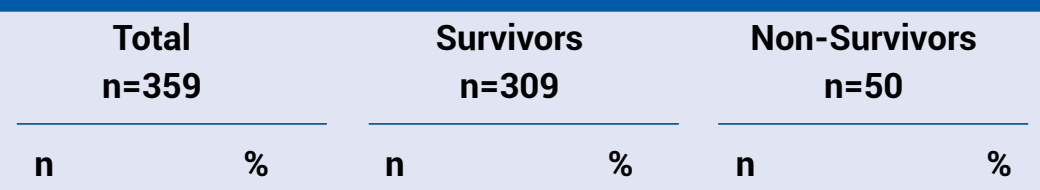

Indication for warfarin

Pulmonary thromboembolism

Valvular heart disease

44

12.5

39

12.8

5

10.4

0.39

Cerebrovascular disease

87

24.6

24.9

22.9

Dysrhythmia

45

12.7

12.8

11

12.5

Venous thromboembolism 18

$45.0 \quad 133$

$43.6 \quad 26$

54.2

leeding localization

Upper gastrointestinal

220

5.1

5.9

0.0

Lower gastrointestinal

139

$61.2 \quad 182$

58.9

38

76.0

0.001 *

Laboratory

International normalized ratio, mean (SD)

Albumin, g/dL, mean (SD)

$9.612(5.287)$

$9.442(5.178)$

$10.660(5.867)$

0.13

$3.61(0.62)$

$3.66(0.57)$

$3.34(0.81)$

$0.002 *$

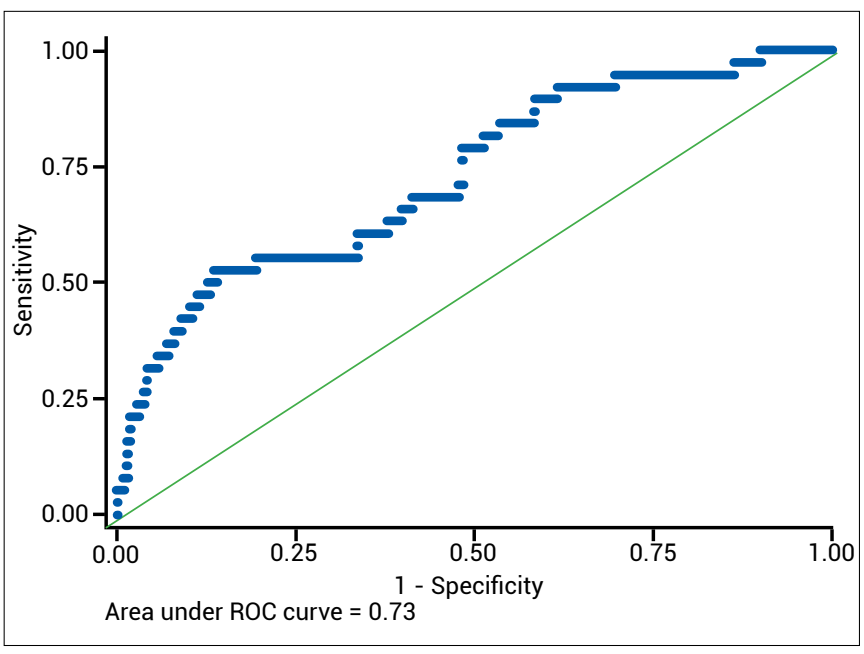

Figure 2. The area of under the curve for significant predictors of 30 -day all-cause mortality.

\section{Discussion}

In the current study, we found that advanced age, hypertension, serum albumin level, and alcohol use were significantly associated with 30-day all-cause mortality in patients admitted with warfarin overdose and GIB. We created a novel nomogram that includes 4 variables with an AUC of 0.73 (acceptable discrimination). As defining predictors for mortality is important in terms of affecting patient follow-up, length of hospital stay, and treatment protocols, our nomogram can be conveniently used to predict individual 30-day all-cause mortality in warfarin

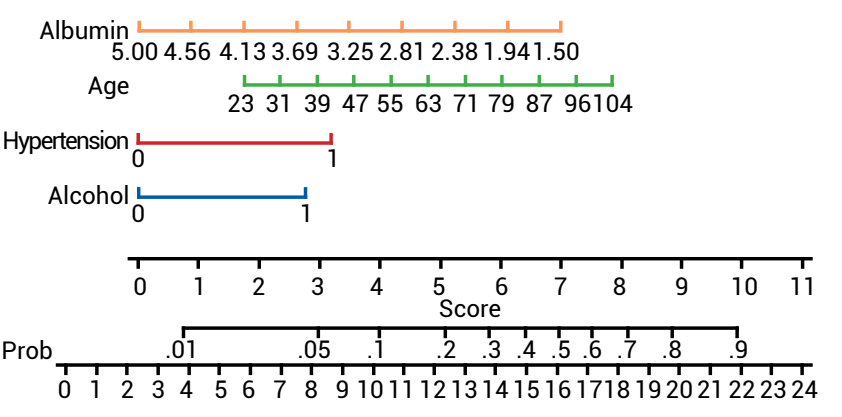

Figure 3. The nomogram for 30-day all-cause mortality.

overdose patients undergoing endoscopy for GIB.

Various scoring systems have been developed to predict the risk of bleeding in patients receiving warfarin therapy: Anticoagulation and risk factors in atrial fibrillation, Outcome Record for Better Informed Treatment scores, HASBLED score (hypertension, abnormal liver or kidney function, stroke, bleeding tendency, and variable INR, age $>65$, drugs, or alcohol) are some of these. ${ }^{[11,12]}$ However, these models are not specifically developed for mortality in GIS bleeding patients. In our model, the discrimination (as assessed by c-index) of the risk score determined according to the nomogram was found to be 0.73. It is thought that it may be beneficial in clinical practice as it had moderate accuracy.

In our study, the short-term 30-day mortality rate was $14 \%$ in patients who underwent endoscopy with the diagnosis 


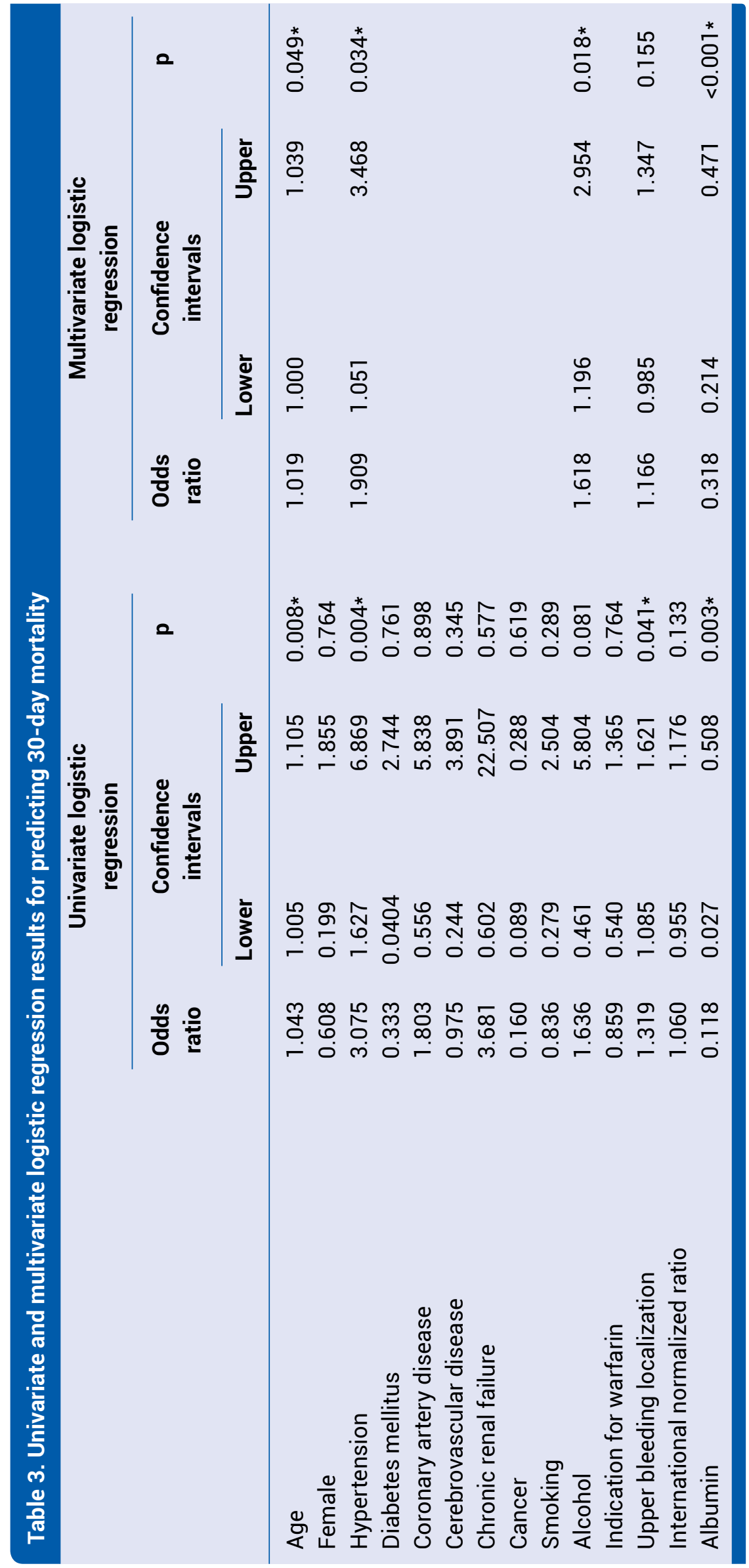

of warfarin overdose and GIB. In another study, mortality was observed in $8.7 \%$ of 172 patients who presented with GIB treated with warfarin during 1-month follow-up. ${ }^{[13]}$ The high mortality rate in our study was thought to be related to the evaluation of a specific group undergoing endoscopic treatment with GIB, and the fact that the selected group included high-risk patients with higher bleeding findings that would require endoscopic treatment.

In a meta-analysis, it was shown that re-initiation of warfarin following discontinuation due to GIB was associated with a decrease in thromboembolic events and mortality without a statistically significant increase in recurrent GIB. ${ }^{[14]}$ In our study, after the bleeding control was achieved, an appropriate anticoagulant regimen was initiated for the patients.

According to our study data, advanced age, hypertension, alcohol use, and serum albumin levels are associated with 30-day mortality in patients who underwent endoscopic treatment with GIB after warfarin overdose. Unlike the HAS-BLED scoring, which is a guide in terms of bleeding risk in the OAC treatment plan, serum albumin level was associated with mortality in our study, while there was no difference between survivors and non-survivors groups in terms of cerebrovascular event history, presence of chronic renal failure, and smoking. It may be considered to be improved by adding serum albumin level parameter to HAS-BLED scoring. In the study of Zhang et al., male gender, coronary heart disease, hypertension, stroke, systolic blood pressure, hematocrit, plasma albumin, and alanine amino transferase levels are associated with GIB in patients applying to cardiology. ${ }^{[15]}$ In a study aimed at assessing the risks 
of re-bleeding and thromboembolism in anticoagulated patients with acute GIB, re-bleeding was associated with low platelet count and albumin level and low dose aspirin use, but the HAS-BLED score, no endoscopic result, heparin bridge, or INR 2.5. ${ }^{[16]}$ Based on these studies and our study data, it should be considered to develop new risk score models including serum albumin level.

Similar studies with new generation OACs and developing mortality determinant nomograms may be a guide in the management of follow-up and treatment of patients with bleeding complications. This article shows the factors predicting 30-day mortality in patients undergoing endoscopic therapy with warfarin overdose and GIB, and provides a practical algorithm to support clinicians in the management of these patients.

There are several limitations of our study. First, because the study was designed retrospectively, parameters affecting long-term mortality could not be evaluated. Second, we were not able to include some other clinical and laboratory parameters due to lack of information. Finally, we were not able to test our model in an external validation cohort which might have strengthened our findings.

\section{Conclusion}

Advanced age, hypertension, serum albumin level, and alcohol use are associated with 30-day all-cause mortality in patients undergoing warfarin overdose and endoscopic treatment with GIB. An easily applicable and relatively cheap nomogram that includes age, hypertension, serum albumin level, and alcohol can be used to define high-risk warfarin overdose patients admitting with GIB.

\section{Disclosures}

Ethichs Committee Approval: The study was approved by the Ankara City Hospital Ethics Committee (date: 04/07/2021, decision no: E2-21-424).

Peer-review: Externally peer-reviewed.

Conflict of Interest: None declared.

Authorship Contributions: Concept - H.T., A.A.S.; Design - H.T., A.A.S.; Supervision - H.T., A.A.S.; Materials - H.T., A.A.S.; Data collection and/or processing - H.T., A.A.S.; Analysis and/ or interpretation - H.T.; Literature search - H.T.; Writing - H.T.; Critical review - H.T., A.A.S.

\section{References}

1. Tomaselli GF, Mahaffey KW, Cuker A, Dobesh PP, Doherty JU, Eikelboom JW, et al. 2020 ACC expert consensus decision pathway on management of bleeding in patients on oral anticoagulants: A report of the American college of cardiology solution set oversight committee. J Am Coll Cardiol 2020;76:594-622. [CrossRef]

2. Levine $M$, Pizon AF, Padilla-Jones A, Ruha AM. Warfarin overdose: A 25-year experience. J Med Toxicol 2014;10:156-64. [CrossRef]

3. Witt DM, Delate T, Garcia D, Clark NP, Hylek EM, Ageno W, et al. Risk of thromboembolism, recurrent hemorrhage, and death after warfarin therapy interruption for gastrointestinal tract bleeding. Arch Intern Med 2012;172:1484-91. [CrossRef]

4. Pautas E, Gouin-Thibault I, Debray M, Gaussem P, Siguret V. Haemorrhagic complications of Vitamin $\mathrm{K}$ antagonists in the elderly: Risk factors and management. Drugs Aging 2006;23:13-25. [CrossRef]

5. Verstuyft C, Robert A, Morin S, Loriot MA, Flahault A, Beaune $P$, et al. Genetic and environmental risk factors for oral anticoagulant overdose. Eur J Clin Pharmacol 2003;58:739-45. [CrossRef]

6. Patel P, Nigam N, Sengupta N. Resumption of warfarin after hospitalization for lower gastrointestinal bleeding and mortality benefits. J Clin Gastroenterol 2018;52:545-50. [CrossRef]

7. Lanas-Gimeno A, Lanas A. Risk of gastrointestinal bleeding during anticoagulant treatment. Expert Opin Drug Saf 2017;16:673-85. [CrossRef]

8. Zhu W, He W, Guo L, Wang X, Hong K. The HAS-BLED score for predicting major bleeding risk in anticoagulated patients with atrial fibrillation: A systematic review and meta-analysis. Clin Cardiol 2015;38:555-61. [CrossRef]

9. Aoki T, Nagata N, Niikura R, Shimbo T, Tanaka S, Sekine K, et al. Recurrence and mortality among patients hospitalized for acute lower gastrointestinal bleeding. Clin Gastroenterol Hepatol 2015;13:488-94.e1. [CrossRef]

10. Gage BF, Eby C, Milligan PE, Banet GA, Duncan JR, McLeod $\mathrm{HL}$. Use of pharmacogenetics and clinical factors to predict the maintenance dose of warfarin. Thromb Haemost 2004;91:87-94. [CrossRef]

11. Lip GY, Skjøth F, Nielsen PB, Kjældgaard JN, Larsen TB. The HAS-BLED, ATRIA, and ORBIT bleeding scores in atrial fibrillation patients using non-Vitamin $\mathrm{K}$ antagonist oral anticoagulants. Am J Med 2018;131:574.e13-27. [CrossRef]

12. O'Brien EC, Simon DN, Thomas LE, Hylek EM, Gersh BJ, Ansell JE, et al. The ORBIT bleeding score: A simple bedside score to assess bleeding risk in atrial fibrillation. Eur Heart $\mathrm{J}$ 2015;36:3258-64. [CrossRef]

13. Turcato G, Bonora A, Zorzi E, Zaboli A, Zannoni M, Ricci G, et al. Thirty-day mortality in atrial fibrillation patients with gastrointestinal bleeding in the emergency department: differences between direct oral anticoagulant and warfarin users. 
Intern Emerg Med 2020;15:311-8. [CrossRef]

14. Chai-Adisaksopha C, Hillis C, Monreal M, Witt DM, Crowther M. Thromboembolic events, recurrent bleeding and mortality after resuming anticoagulant following gastrointestinal bleeding. A meta-analysis. Thromb Haemost 2015;114:819-25. [CrossRef]

15. Zhang M, Liu D, Wang Q, Geng X, Hou Q, Gu G, et al. Gas- trointestinal bleeding in patients admitted to cardiology: risk factors and a new risk score. Hellenic $\mathrm{J}$ Cardiol 2020;2020:30163-9. [CrossRef]

16. Nagata N, Sakurai T, Moriyasu S, Shimbo T, Okubo H, Watanabe $\mathrm{K}$, et al. Impact of INR monitoring, reversal agent use, heparin bridging, and anticoagulant interruption on rebleeding and thromboembolism in acute gastrointestinal bleeding. PLoS One 2017;12:e0183423. [CrossRef] 